\title{
Palatoradicular Groove Associated Defect Resolved with GIC, Alloplastic Graft and GTR Membrane
}

\author{
${ }^{1}$ CG Devaraj, ${ }^{2}$ Ashish Yadav, ${ }^{3}$ Swati Sharma, ${ }^{4}$ Meenakshi Meena, ${ }^{5}$ Pulak Mishra, ${ }^{6}$ Sonia Godara, ${ }^{7}$ Radhika Kapoor
}

\begin{abstract}
The region of maxillary lateral incisor is an area of embryological importance with major and minor malformations like, gemination, fusion, cysts, dens in dente, peg-shaped laterals, talon cusps, supernumerary roots, and palatogingival groove (PGG). Palatogingival groove is a developmental groove usually found on the lingual aspect of maxillary incisors. It can be simple or complex on the basis of its connection to the pulp. These grooves act as a nidus for plaque accumulation which destroys the sulcular epithelium and later deeper parts of the periodontium. Since proper cleaning of this region is difficult by patient himself, it can lead to serious localized periodontal lesion or can communicate with endodontic lesion. Suggested treatment modalities are curettage of the affected tissues, elimination of the groove by grinding (saucerization), or by sealing with a variety of filling materials. If the groove extends beyond the middle-third of the root apex, surgical procedures are required, including use of barriers and intraosseous alloplastic graft to correct the defect.
\end{abstract}

Keywords: Developmental groove, Maxillary incisors, Palatogingival groove, Saucerization.

How to cite this article: Devaraj CG, Yadav A, Sharma S, Meena M, Mishra P, Godara S, Kapoor R. PalatoRadicular Groove Associated Defect Resolved with GIC, Alloplastic Graft and GTR Membrane. J Mahatma Gandhi Univ Med Sci Tech 2016;1(2):75-78.

Source of support: Nil

Conflict of interest: None

\section{INTRODUCTION}

Anatomic aberrations are often seen in the human dentition. Aberrations affecting the external and internal morphology of the tooth can at times be the cause of complex pathological conditions involving the pulpal and periodontal tissues and can pose a challenge to the clinician for diagnosis and clinical management. ${ }^{1}$

The region of maxillary lateral incisor is an area of embryological importance. A great number of

\footnotetext{
${ }^{1}$ Professor and Head, ${ }^{2,3}$ Reader, ${ }^{4}$ Senior Lecturer

${ }^{5-7}$ Postgraduate Student

${ }^{1-7}$ Department of Periodontology, Mahatma Gandhi Dental College and Hospital, Jaipur, Rajasthan, India

Corresponding Author: CG Devaraj, Professor and Head Department of Periodontology, Mahatma Gandhi Dental College and Hospital, Jaipur, Rajasthan, India, email: dr_devarajcg@ yahoo.com
}

major and minor malformations occur in this area; for instance gemination, fusion, cysts, dens in dente, pegshaped laterals, talon cusps, supernumerary roots, and palatogingival groove (PGG). Palatogingival groove is defined as "a developmental groove in a root that, when present, is usually found on the lingual aspect of maxillary incisor teeth". Palatogingival grooves are also called as palatoradicular groove, radicular anomaly, distolingual groove, and radicular lingual groove. ${ }^{1}$ The groove was first mentioned in a dental anatomy text in 1917, later described by Zeisz and Nuckolin in $1949 .^{2}$ Kovacs in 1971 called it as a 'syndesmo-corono radicular' tooth. ${ }^{3}$ It may result from an infolding of the enamel organ and Hertwigs epithelial root sheath (HERS). ${ }^{4}$ Some researchers have also suggested that the anomaly results from an attempt to form another root. ${ }^{5}$

Goon et $\mathrm{al}^{6}$ suggested a classification, which represents two types of PGG, simple and complex. The simple PGG does not communicate with the pulp and represents a partial infolding of Hertwig's epithelial root sheath (HERS), while complex PGG communicate directly with the pulp and groove that extend the length of the root. In rare cases, the groove may lead to minor accessory root, which may contain a root canal. ${ }^{7}$

These grooves act as a nidus for plaque accumulation which destroys the sulcular epithelium and later deeper parts of the periodontium. Since proper cleaning of the site is difficult, if not impossible for the patient, it finally results in the formation of a severe localized periodontal lesion. These grooves if extending to the root apex may also lead to combined endodontic-periodontal lesions, since there is evidence of communication between the pulp canal system and the periodontium through accessory canals. The prognosis of the tooth presenting with a palatogingival groove depends upon the extension of the groove in the apical area. Shallow grooves are amenable to correction by odontoplasty in association with periodontal treatment. However, when the groove is more advanced, treatment of the tooth becomes complicated. ${ }^{8}$ Suggested treatment modalities were curettage of the affected tissues, elimination of the groove by grinding (saucerization), or by sealing with a variety of filling materials. If the groove extends beyond the middle-third of the root apex, surgical procedures 


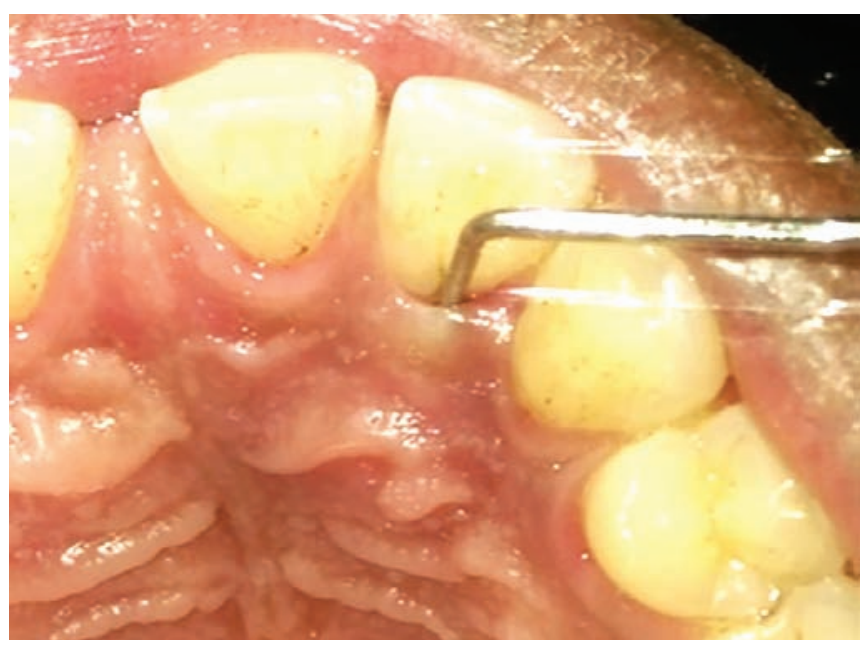

Fig. 1: Periodontal probing showing deep pocket on palatal aspect

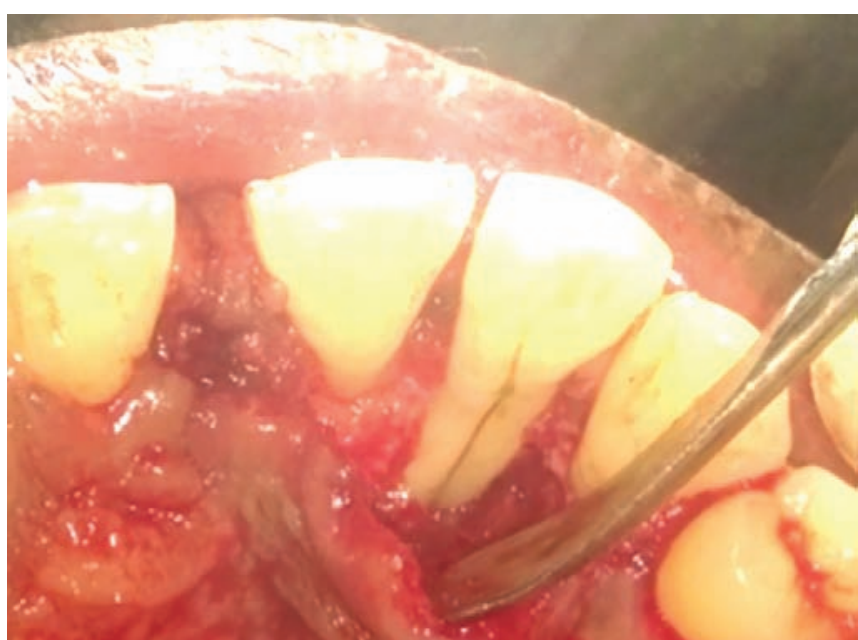

Fig. 3: Palatal flap raised showing the palatoradicular groove

are required, including use of barriers and intraosseous alloplastic graft to correct the defect.

Presented is a case of a PGG in a maxillary incisor treated successfully with a restoration of the groove with Glass Ionomer Cement (GIC), followed by alloplastic bone graft and guided tissue regeneration (GTR) membrane.

\section{CASE REPORT}

A 24-year-old patient reported to the Department of Periodontology and Implantology, Mahatma Gandhi Dental College and Hospital, Jaipur, with the chief complaint of pus exudation and persistent dull pain in upper right front teeth region for the last 4 months. On examination, there was pus exudation from the gingival sulcus of the palatal aspect of maxillary right lateral incisor.

On careful periodontal probing, a deep groove extending from the cingulum to the mid root area was

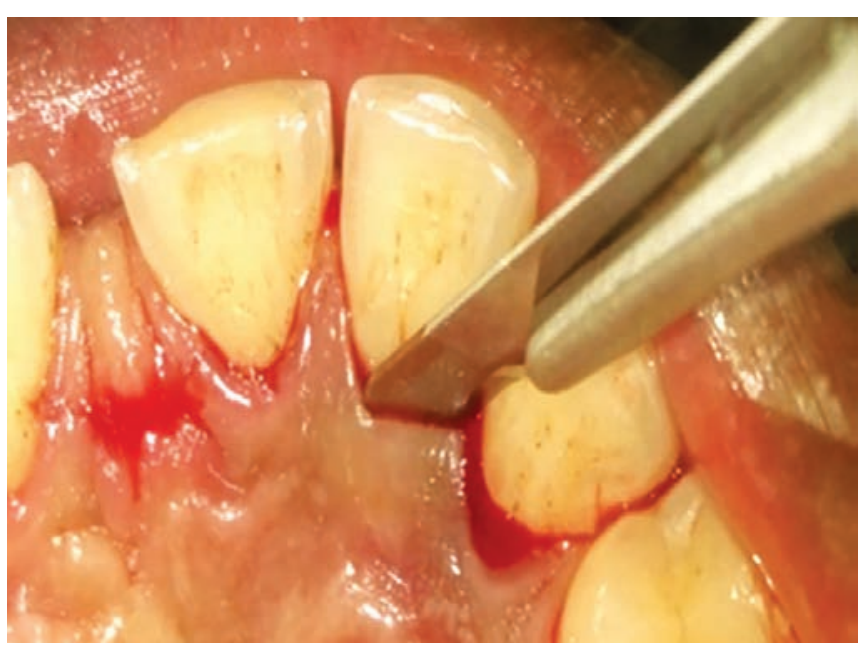

Fig. 2: Depicting intrasulcular incision on palatal aspect

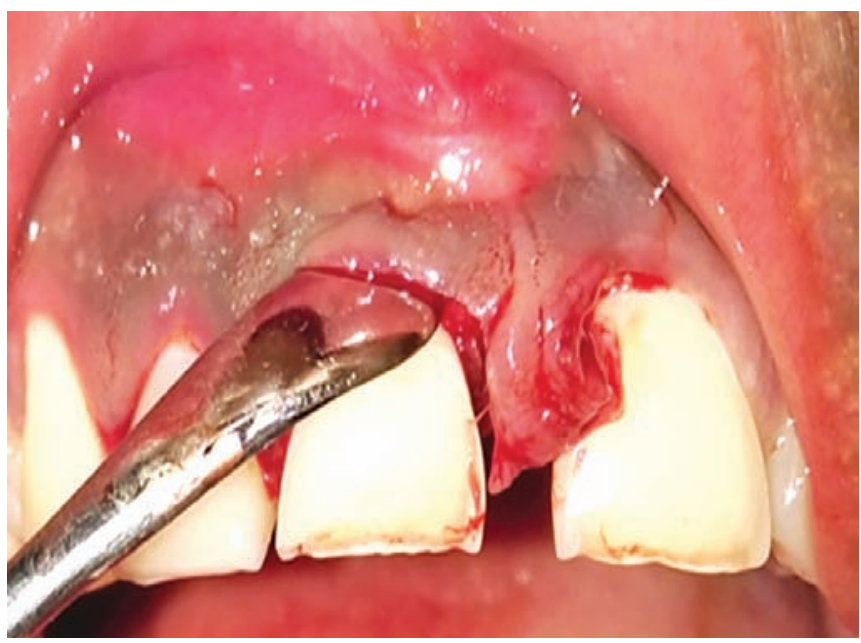

Fig. 4: Papilla preservation flap

noted (Fig. 1). Radiographic examination with a radioopaque material placed into the groove gave the exact length, apical location and termination of the palatoradicular groove. Further radiographic examination revealed extensive alveolar bone loss in relation maxillary lateral incisor.

To eliminate the radicular groove and promote periodontal regeneration, surgical periodontal treatment was planned. The area was anesthetized and mucoperiosteal flaps on both labial and palatal aspects were elevated. As the patient had spacing between the central incisors, a papilla preservation flap was also raised (Figs 2 to 4). Thorough degranulation of the lesion was performed. An advanced circumferential bony defect was found after degranulation. The palatal radicular groove was eliminated by means of saucerization using a round bur (Fig. 5). After the saucerization of groove was completed, it was felt necessary to condition and seal with GIC. 


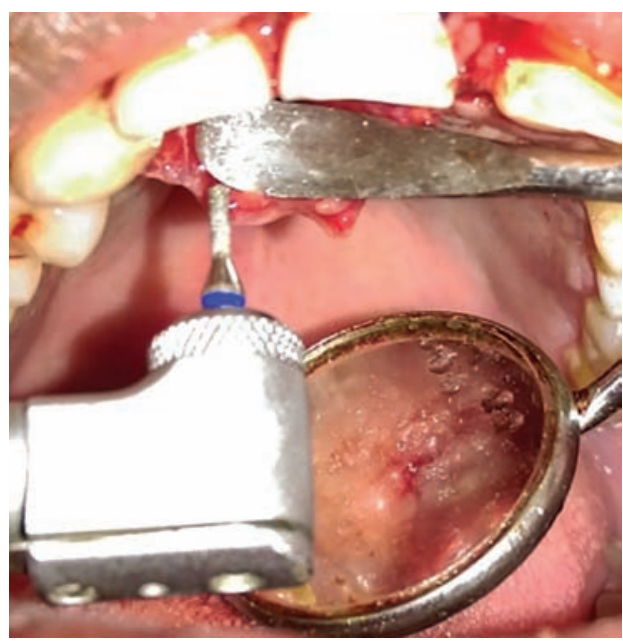

Fig. 5: Radiculoplasty to eliminate the palatogingival groove

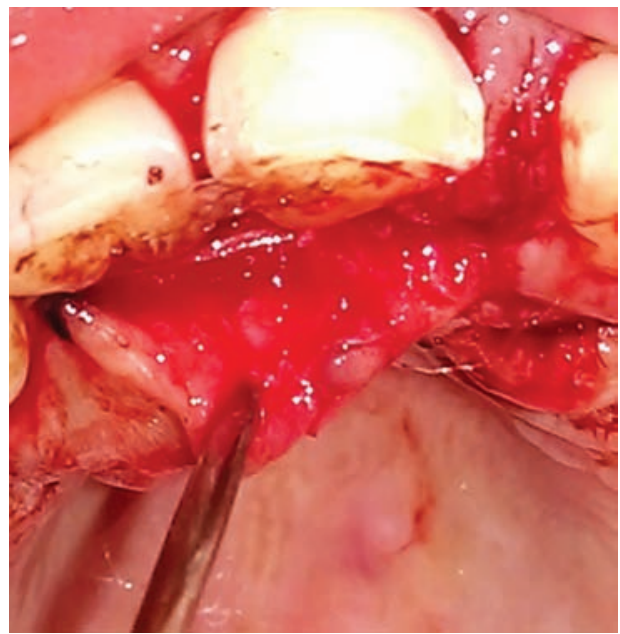

Fig. 7: GTR membrane placed

\section{ALLOPLASTIC BONE GRAFT AND GTR MEMBRANE PLACEMENT}

The flap was readapted, and a holding suture was placed and stabilized with nonabsorbable silk sutures, on mesial and distal surface of right central incisor (Fig. 6). The knot was not tightened to allow placement of alloplastic bone graft and to prevent the graft material from getting displaced. Hydroxyapatite graft material was mixed with saline and placed into the deep bony defect. It was followed by placement of GTR membrane (Heli guide) to promote periodontal regeneration and to avoid formation of long junctional epithelium (Fig. 7).

After flap closure, the surgical area was covered with a non-eugenol periodontal dressing (Coe pak). The patient was prescribed antibiotics and non steroidal drugs for a week. The patient was asked to rinse his mouth with chlorhexidine mouth rinse $(0.2 \%)$ twice a day for a week for chemical plaque control measure. At 1 week post surgery follow-up, the periodontal pack and sutures were removed. The patient was recalled at regular intervals for

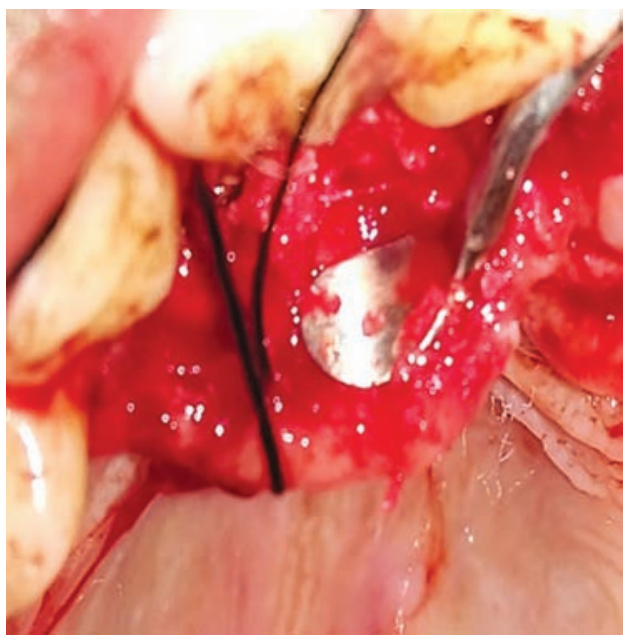

Fig. 6: Holding sutures and GIC, alloplastic bone graft

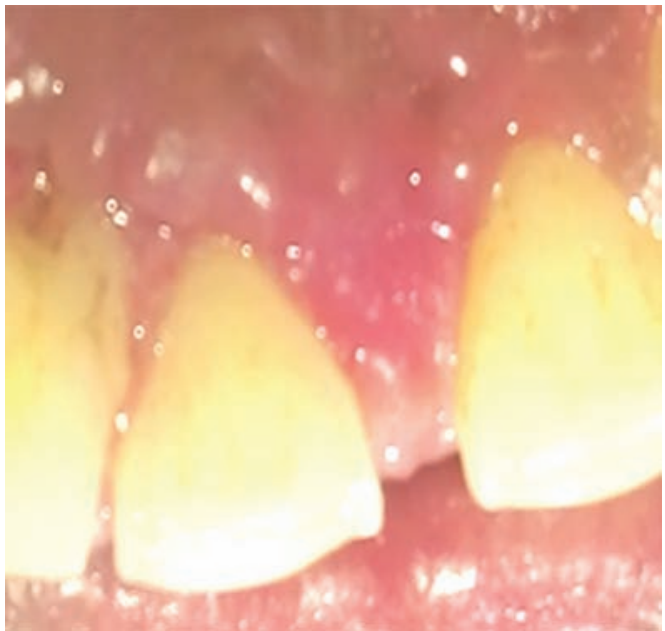

Fig. 8: Postoperative follow-up after 3 months

follow-up (Fig. 8). After 3 months, the probing depth had reduced from 8 to $3 \mathrm{~mm}$ and the area was periodontally healthy.

\section{DISCUSSION}

Palatogingival groove is mostly found in maxillary lateral incisors and have been implicated as an initiating factor in localized gingivitis and periodontitis. Focal loss of periodontal attachment associated with these grooves, some of which may extend to the apical third of the root could result in a hopeless prognosis for tooth retention. In cases where the pulp has also become necrotic, the tooth requires endodontic treatment in addition to periodontal therapy. Albaricci et al studied the prevalence and different morphological conditions of the palate-radicular grooves in 376 maxillary lateral and central incisors. Prevalence in lateral incisor was $11.1 \%$, only $8.6 \%$ of PGG reached the root apex, while $97.1 \%$ were considered to be flat $(<1 \mathrm{~mm})^{9}$ 
Kogan in 1986 examined 3168 extracted maxillary central and lateral incisors. Palatogingival grooves found on $4.6 \%$ of maxillary incisors (3.4\% maxillary centrals and $5.6 \%$ on maxillary lateral incisors), $54 \%$ of palatogingival grooves terminated on the root with $43 \%$ of those extending less than $5 \mathrm{~mm}$ and $47 \%$ extending 6 to $10 \mathrm{~mm} \cdot{ }^{10}$ A genetic mechanism and racial link has also been proposed. The incidence of radicular groove is reported to be between 2.8 and $18 \%{ }^{11}$

The predominance of palatogingival groove in maxillary lateral incisor is due to an undesirable position of the lateral incisor during the period of maxillary growth. The tooth, although till a tooth germ, becomes surrounded by the central incisors, canine and first premolar that are in a more advanced phase of dental development. Mineralization of the crown of the maxillary lateral incisor starts later compared with other teeth, making this tooth germ, under these conditions highly susceptible to folding. Accessory canals connecting to the pulp in the depth of grooves could lead to bacterial ingress to the pulp space. ${ }^{12}$

The prognosis of a tooth with a palatoradicular groove depends mainly on the location and extension of the groove. When the PGG is shallow, the prognosis can be estimated as good since there will be neither a deep bony defect nor pulpal damage. Successful treatment of the PGG depends on the ability to eradicate inflammatory irritants, by eliminating the groove. Radiculopathy is recommended to eliminate the groove, which often harbors bacteria and debris leading to a localized inflammatory reaction..$^{13}$ The grooves can be eliminated by odontoplasty in the case of shallow ones or by restoring with amalgam, composite or GICs. In the present case, saucerization of the groove was carried out followed by restoration with GIC. To provide a scaffold for regeneration of the bone defect around the tooth, alloplastic bone graft with GTR membrane were used.

\section{CONCLUSION}

Palatoradicular grooves are a unique deformity in the process of tooth development. As they are not visible clinically, it has always been a dilemma while diagnosing a case of recurrent abscess or bone loss localized to a single tooth. A proper diagnostic procedure and an adequate treatment planning are necessary for preventing any recurrence of the localized condition. Its treatment involves multidisciplinary approach.

\section{REFERENCES}

1. Dharamsi A, Garhnayak L, Garhnayak M, Singh GP, Chaturvedy V. Comprehensive management of a lateral incisor with a deep palatoradicular groove: a case report. Indian J Stomatol 2011;2(2):144-147.

2. Zeisz RC, Nuckolin J. Dental Anatomy. St Louis, CV Mosby Co 1949;65.

3. Kovacs J. A systematic description of dental roots. Dahlberg AA. Dental morphology and evolution. University of Chicago Press, Chicago, 1971:211-256.

4. Shalu JP. Palatogingival developmental groove. Quintessence Int 2001;32:349-351.

5. Lee KW, Lee EC, Poon KY. Palatogingival grooves in maxillary incisors. British Dental J 1968;124:14-18.

6. Goon WW, Carpenter WM, Brace NM, Ahlfeld RJ. Complex facial radicular groove in a maxillary lateral incisor. J Endod 1991;17:244-248.

7. Dexton AJ, Arundas D, Rameshkumar M, Shoba K. Retreatodontics in maxillary lateral incisor with supernumerary root. J Conserv Dent 2011;14:322-324.

8. Cohen E. Cosmetic and reconstructive periodontal surgery, 3rd ed, p.103.

9. Albaricci MF, de Toledo BE, Zuza EP, Gomes DA, Rosetti EP. Prevalence and features of palate-radicular grooves: an in vitro study. Int Acad Periodontol 2008;10:2-5.

10. Kogan SL. The prevalence, location and conformation of palatoradicular grooves in maxillary incisors. J Periodontol 1986;57:2312.

11. Hou GL, Tsai CC. Relationship between palatoradicular grooves and localized periodontitis. J Clini Periodontol 1993;20:678-682.

12. Rethman MP. Treatment of a palatogingival groove using enamel matrix derivative. Compend Contin Educ Dent 2001;22:792-797.

13. Zuccheli G, Mele M, Chechi L. The papilla amplification flap for the treatment of a localized periodontal defects associated with a palatal groove. J Periodontol 2006;77:1788-1796. 